\title{
BOREL LIFTINGS OF THE MEASURE ALGEBRA AND THE FAILURE OF THE CONTINUUM HYPOTHESIS
}

\author{
T. CARLSON, R. FRANKIEWICZ, AND P. ZBIERSKI
}

(Communicated by Andreas R. Blass)

\begin{abstract}
It is proved that the failure of the continuum hypothesis is consistent with the existence of a Borel lifting for the Lebesgue measure algebra and an embedding of the Lebesgue measure algebra into $\wp(\omega) /$ finite.
\end{abstract}

Let $L M$ denote the field of Lebesgue measurable sets and let $\Delta$ be the ideal of measure zero sets. A (measurable) lifting of the measure algebra $L M / \Delta$ is any Boolean embedding $l: L M / \Delta \rightarrow L M$ such that $l(\mathbf{a}) \in \mathbf{a}$ for all $\mathbf{a}$ in $L M / \Delta$. If, in addition, each $l(a)$ is a Borel set, then $l$ is called a Borel lifting. It is known (see [M]) that each measure algebra of a $\sigma$-finite measure has a measurable lifting. In [N-S] it is proved that $\mathrm{CH}$ (the continuum hypothesis) implies that $L M / \Delta$ has a Borel lifting. On the other hand, Shelah proved in [S] that $2^{\omega}=\omega_{2}$ is consistent with the nonexistence of Borel liftings of $L M / \Delta$.

Our first result is that $2^{\omega}=\omega_{2}$ is consistent with the existence of a Borel lifting of $L M / \Delta$. Hence, the existence of a Borel lifting of $L M / \Delta$ is not equivalent to $\mathrm{CH}$. This result was originally proved more than ten years ago by the first author. The proof was recently rediscovered by the other two authors after proving, by a similar argument, our second result: $2^{\omega}=\omega_{2}$ is consistent with the existence of an embedding of $L M / \Delta$ into $\wp(\omega) /$ finite.

If $\mathbf{B}$ is a subalgebra of $L M / \Delta$ and $\mathbf{b}$ is an element of $L M / \Delta$, then the gap determined by $\mathbf{b}$ over $\mathbf{B}$ is the pair $\langle C, D\rangle$, where $C$ consists of all elements of $\mathbf{B}$ with $\mathbf{c} \leq \mathbf{b}$ and $D$ consists of all elements $\mathbf{d}$ of $\mathbf{B}$ with $\mathbf{b} \leq \mathbf{d} .\langle C, D\rangle$ is said to be countably generated if $C$ is countably generated as an ideal and $D$ is countably generated as a filter (possibly improper).

Whenever $M$ and $N$ are inner models of ZFC with $M \subseteq N$ we will identify $(L M / \Delta)^{M}$ with a subalgebra of $(L M / \Delta)^{N}$ via the natural embedding.

Lemma 1. Let $Q$ be the partial ordering for adding a Cohen real. In $V^{Q}$ the gap determined by any element of $L M / \Delta$ over $(L M / \Delta)^{V}$ is countably generated.

Proof. We will argue in $V Q$. Let $G$ be the $V$-generic filter over $Q$ and suppose that $\mathbf{b} \in L M / \Delta$. Fix a name $\underline{\mathbf{b}}$ for $\mathbf{b}$. For each $q \in G$ define $\mathbf{b}_{q}$ to be the supremum of all $\mathbf{c}$ in $(L M / \Delta)^{V}$ such that $q$ forces $\mathbf{c} \leq \underline{\mathbf{b}}$. The collection of

Received by the editors July 2, 1991 and, in revised form, July 16, 1992.

1991 Mathematics Subject Classification. Primary 03E05, 03E15; Secondary 03E35, 03 E50.

The first author was supported by the NSF. 
$\mathbf{b}_{q}$ generates the first component of the gap determined by $\mathbf{b}$. Similarly, the second component of the gap is countably generated.

Lemma 2. Suppose $P$ is the partial ordering for adding $\kappa$ Cohen reals for some cardinal $\kappa$. In $V^{P}$ the gap of $\mathbf{b}$ over $(L M / \Delta)^{V}$ is countably generated for every $\mathbf{b} \in L M / \Delta$.

Proof. This follows from Lemma 1 since in $V^{P}$ every element of $L M / \Delta$ is in $V[x]$ for some Cohen real $x$.

Lemma 3. Assume B is a subalgebra of $L M / \Delta$ such that the gap determined by $\mathbf{b}$ over $\mathbf{B}$ is countably generated for each $\mathbf{b} \in L M / \Delta$. If $\mathbf{C}$ is the subalgebra generated by $\mathbf{B}$ along with some countable set $C$, then the gap determined by b over $\mathbf{C}$ is countably generated for each $\mathbf{b} \in L M / \Delta$.

Proof. Note that the lower part of the gap determined by $\mathbf{b}$ over $\mathbf{C}$ is generated by all $\mathbf{b}^{\prime} \wedge \mathbf{c}$, where $\mathbf{c}$ is in the subalgebra generated by $C$ and $\mathbf{b}^{\prime}$ is in the lower part of the gap determined by $\mathbf{b} \vee(-\mathbf{c})$. Since the collection of such $\mathbf{b}^{\prime}$ is countably generated, so also is the lower part of the gap determined by $\mathbf{b}$ over C. Similarly, the upper part of the gap determined by b over $\mathbf{C}$ is countably generated.

Lemma 4. Assume CH. If $P$ is the partial ordering for adding $\omega_{2}$ Cohen reals, then in $V^{P}$ there is an enumeration of $L M / \Delta$ of length $\omega_{2}$ such that the gap determined by each element over the subalgebra generated by the previous elements is countably generated.

Proof. We will argue in $V^{P}$. Let $\mathbf{B}_{\alpha}$ be the measure algebra in the extension of $V$ by the first $\alpha$ Cohen reals. For $\alpha<\omega_{2}, \mathbf{B}_{\alpha}$ has size $\omega_{1}$. Enumerate $L M / \Delta$ in a sequence $\mathbf{b}_{\beta}\left(\beta \in \omega_{2}\right)$ such that $\mathbf{B}_{\alpha}$ is enumerated in the $\alpha$ th interval of length $\omega_{1}$, i.e., $\mathbf{b}_{\beta}\left(\omega_{1} \alpha \leq \beta<\omega_{1}(\alpha+1)\right)$ enumerates $\mathbf{B}_{\alpha}$.

Suppose that $\beta<\omega_{2}$ and let $\mathbf{C}$ be the algebra generated by the set of $\mathbf{b}_{\gamma}$ with $\gamma<\beta$. We must show that the gap determined by $\mathbf{b}_{\beta}$ over $\mathbf{C}$ is countably generated. There are $\alpha<\omega_{2}$ and $\xi<\omega_{1}$ such that $\beta=\omega_{1} \alpha+\xi$. Let $\mathbf{B}$ be the algebra generated by the $\mathbf{b}_{\gamma}$ with $\gamma<\omega_{1} \alpha$. By Lemma 3, it suffices to show that the gap determined by $\mathbf{b}$ over $\mathbf{B}$ is countably generated for each $\mathbf{b}$ in $L M / \Delta$. The case $\alpha=0$ is clear. Notice that by Lemma 2, for any $\delta$ each element of $L M / \Delta$ determines a gap over $\mathbf{B}_{\delta}$ which is countably generated. Hence if $\alpha$ has cofinality $\omega_{1}$ or is a successor ordinal, then we are done since $\mathbf{B}$ is $\mathbf{B}_{\alpha}$ and $\mathbf{B}_{\alpha-1}$, respectively. If $\alpha$ has cofinality $\omega$, then $\mathbf{B}$ is $\bigcup\left\{\mathbf{B}_{\delta}: \delta<\alpha\right\}$ and the gap determined by $\mathbf{b}$ over $\mathbf{B}$ is just the union of the gaps determined by $\mathbf{b}$ over $\mathbf{B}_{\delta}$ for $\delta<\alpha$, each of which is countably generated. Hence the gap of $\mathbf{b}$ over $\mathbf{B}$ is countably generated.

Lemma 5. If there is an enumeration of $L M / \Delta$ in which the gap determined by any element in the enumeration over the subalgebra generated by its predecessors is countably generated, then there is a Borel lifting.

Proof. Suppose $\mathbf{b}_{\beta}(\beta \in \kappa)$ is an enumeration as described in the hypothesis. Let $\mathbf{B}_{\alpha}$ be the algebra generated by the $\mathbf{b}_{\beta}$ with $\beta<\alpha$. Define a continuous tower of partial liftings $h_{\alpha}$ on $\mathbf{B}_{\alpha}$ by induction on $\alpha$. By Sikorski's theorem on extending homomorphisms, we can extend $h_{\alpha}$ to $\mathbf{b}_{\alpha}$ provided we can find a Borel set $X$ in $\mathbf{b}_{\alpha}$ such that $h_{\alpha}(\mathbf{c}) \subseteq X \subseteq h_{\alpha}(\mathbf{d})$ for all $\mathbf{c} \in C$ and $\mathbf{d} \in D$ 
where $\langle C, D\rangle$ is the gap determined by $\mathbf{b}_{\alpha}$ over $\mathbf{B}_{\alpha}$. This can be done since $\langle C, D\rangle$ is countably generated.

Theorem 1. Assume CH. If $P$ is the partial ordering for adding $\omega_{2}$ Cohen reals, then in $V^{P}$ there is a Borel lifting of $L M / \Delta$. In fact, every Borel lifting in $V$ extends to a Borel lifting in $V^{P}$.

Proof. The existence of a Borel lifting in $V^{P}$ follows immediately from Lemmas 4 and 5. To see that every lifting in $V$ extends to one in $V^{P}$ requires simple modifications to Lemmas 4 and 5 which we leave to the reader.

Now let us consider Boolean embeddings into the algebra $\wp(\omega) /$ finite. If $\mathrm{CH}$ is assumed, then the measure algebra $L M / \Delta$, as well as any algebra of cardinality at most $2^{\omega}$, can be embedded into $\wp(\omega) /$ finite by a well-known theorem of Parovicenko. On the other hand (see $[\mathrm{F}]$ ), it is consistent with $2^{\omega}=\omega_{2}$ that $L M / \Delta$ has no such embedding.

We will use a partial order defined by Kunen for filling a gap in $\wp(\omega) /$ finite. Suppose $\langle C, D\rangle$ is a gap in $\wp(\omega) /$ finite. Let $P(C, D)$ be the partial order consisting of all partial functions $p$ from $\omega$ to 2 such that $\{n: p(n)=1\} /$ finite is in the ideal generated by $C$ and $\{n: p(n) \neq 0\} /$ finite is in the filter generated by $D$. If $\langle C, D\rangle$ is countably generated, then $P(C, D)$ has a countable dense set and a Cohen real will add a generic filter for $P(C, D)$. If $A$ is the subset of $\omega$ added by $P(C, D)$, then $A$ /finite clearly fills the gap $\langle C, D\rangle$.

Theorem 2. Assume CH. If $P$ is the partial ordering for adding $\omega_{2}$ Cohen reals, then in $V^{P}$ there is an embedding of $L M / \Delta$ into $\wp(\omega) /$ finite. In fact, every such embedding in $V$ extends to an embedding in $V^{P}$.

Sketch of proof. We work in $V^{P}$. By Lemma 4, let $\mathbf{b}_{\alpha}\left(\alpha \in \omega_{2}\right)$ be an enumeration of $L M / \Delta$ such that for each $\alpha$ the gap determined by $\mathbf{b}_{\alpha}$ in the subalgebra generated by the $\mathbf{b}_{\beta}$ with $\beta<\alpha$ is countably generated. Let $\mathbf{B}_{\alpha}$ be the algebra generated by the $\mathbf{b}_{\beta}$ with $\beta<\alpha$. Define a continuous tower of embeddings $h_{\alpha}$ of $\mathbf{B}_{\alpha}$ into $\wp(\omega) /$ finite by induction on $\alpha$. In order to construct $h_{\alpha+1}$ from $h_{\alpha}$ we must determine $h_{\alpha+1}\left(\mathbf{b}_{\alpha}\right)$. Routine arguments show that there is a collection $\mathscr{A}$ of at most $\omega_{1}$ dense subsets of $P(C, D)$ such that any filter over $P(C, D)$ which meets all dense sets in $\mathscr{A}$ will determine a subset $A$ of $\omega$ such that we can set $h_{\alpha+1}\left(\mathbf{b}_{\alpha}\right)=A /$ finite. As remarked earlier, the fact that $C$ and $D$ are countably generated means that $P(C, D)$ has a countable dense set. So either $P(C, D)$ has an atom or it is equivalent to the forcing for adding a Cohen real. Standard arguments show that there is a filter which meets any given collection of at most $\omega_{1}$ dense sets in the partial ordering for adding a Cohen real. Therefore, we can find a filter which meets each element of $\mathscr{A}$.

We leave to the reader the modifications needed to construct an embedding extending a given ground model embedding.

Both the theorems can be extended to the algebra of sets with the property of Baire modulo the meager sets.

Problem. Is the existence of a Borel lifting of $L M / \Delta$ equivalent to the existence of an embedding of $L M / \Delta$ into $P(\omega) /$ finite?

Corollary. $2^{\omega}=\omega_{2}$ is consistent with the existence of an isometric embedding of $L^{\infty}$ into $l^{\infty} / c_{0}$. 
Proof. By Theorem 2 we may assume $2^{\omega}=\omega_{2}$ and there is an embedding of $L M / \Delta$ into $\wp(\omega) /$ finite. Let $X$ be the Stone space of $L M / \Delta$. The embedding induces a continuous function from $\omega^{*}$ onto $X$ which in turn induces an isometric embedding of $C(X)$ into $C\left(\omega^{*}\right)$. This implies the corollary since $C(X)$ is isomorphic to $L^{\infty}$ and $C\left(\omega^{*}\right)$ is isomorphic to $l^{\infty} / c_{0}$.

Remark 1. The proof of Theorem 2 can be strengthened so that the embedding $h$ of $L M / \Delta$ into $\wp(\omega) /$ finite has the property that the measure of $\mathbf{b}$ is always the density of $h(\mathbf{b})$.

Remark 2. Theorems 1 and 2 also hold when random reals, rather than Cohen reals, are added to a model of $\mathrm{CH}$.

\section{REFERENCES}

[F] R. Frankiewicz, Some remarks on embeddings of Boolean algebras and topological spaces. II, Fund. Math. 126 (1985), 63-68.

[M] D. Maharam, On a theorem of von Neumann, Proc. Amer. Math. Soc. 9 (1958), 987-994.

[N-S] J. von Neumann and M. H. Stone, The determination of representative elements in the residual classes of a Boolean algebra, Fund. Math. 25 (1935), 353-378.

[S] S. Shelah, Lifting problem of the measure algebra, Israel J. Math. 45 (1983).

Department of Mathematics, Ohio State University, Columbus, Ohio 43210

Institute of Mathematics of the Polish Academy of Sciences, Sniadeckich 8, 00-950 WARSAW, Poland

Department of Mathematics, Warsaw University, Banacha 2, 00-901 Warsaw, Poland 